\title{
ESTUDO DA INFLUÊNCIA DA MARÉ SOB A REMOBILIZAÇÃO DO MATERIAL EM SUSPENSÃO NO PERÍODO DE ENCHENTE NO CANAL DO PORTO DA BARRA GRANDE, ICAPUÍ- CE
}

\author{
Guilherme Augusto Mendonça Maia ${ }^{1}$; \\ Ian Cerdeira de Oliveira Souza ${ }^{2}$; \\ João Capistrano Abreu Neto'; \\ George Satander Sá Freire'; \\ 10.18190/1980-8208/estudosgeologicos.v29n2p54-62 \\ Matheus Edson Mendes Medeiros ${ }^{1}$ \\ ${ }^{1}$ Universidade Federal do Ceará - Fortaleza, Brasil. guilherme.maiageo@gmail.com; \\ joaoabreuneto@gmail.com; satanderfreire@gmail.com; matheunh@ hotmail.com \\ ${ }^{2}$ Universidade Estadual de Campinas, Instituto de Geociências, Programa de Pós- \\ Graduação em Geociências - Campinas, SP. Brasil. cerdeiraian@ gmail.com
}

\section{RESUMO}

Os canais de maré são classificados como áreas que possuem a maré como principal agente de transporte. Diante ao fluxo bidirecional, presente ao longo do canal, são formados bancos arenosos não vegetados circundados, normalmente, por planícies lamosas vegetadas, onde, ocasionalmente o tráfego hidroviário pode ser interrompido, devido ao acúmulo de sedimentos ao redor dos bancos. O Porto da Barra Grande, localizado no município de Icapuí, extremo leste cearense, é utilizado pela comunidade local como estaleiro e ancoradouro para os barcos pesqueiros da região, margeado por mangues e salinas a área apresenta atividade intensas. A economia local está fortemente ligada a atividade pesqueira, que vem sendo prejudicada pelo progressivo assoreamento do canal, dificultando a navegação dos barcos. Dessa maneira, essa pesquisa teve como objetivo analisar e avaliar a influência da maré de enchente na taxa de sedimentação e erosão dentro do canal, a fim de auxiliar nas políticas de gestão, levantando aspectos como velocidade e direção da corrente, material em suspensão e morfologia de seções do canal. Para isso, realizou-se medições no canal em um turno de seis horas consecutivas durante o período de enchente, com coletas de amostras de material em suspensão, leituras regulares do nível de profundidade do canal e aquisição de dados correntométricos. Diante às aquisições e observações em campo, calculou-se a vazão líquida e sólida do canal correspondente ao período, demonstrando a intensa dinâmica de transporte dos sedimentos em suspensão no canal. Através dos resultados comprovou-se a alta dinâmica sedimentar do material em suspensão e caracterizou-se o comportamento hidrodinâmico local sob influência da maré. Dessa forma, os resultados auxiliam em pesquisas para o gerenciamento de obras costeiras futuras, com a finalidade de sanar o contínuo assoreamento do canal que impacta diretamente às atividades econômicas e sociais da região.

Palavras chave: Dinâmica Costeira, Canal de Maré; Correntometria; Material em Suspensão, Icapuí 


\begin{abstract}
Tidal channels are areas that the main transport agent is the tidal. Facing the bidirectional flow along the canal, non-vegetated sandy banks are formed, usually surrounded by vegetated mudflats, where occasionally the waterway traffic may be interrupted due to sediment accumulation around the banks. The Barra Grande Port, located in the city of Icapuí, Far East of Ceará, is used by the local community as a shipyard and anchorage for fishing boats, bordered by mangroves and salt marshes. The fishing activity is one of the principal pillars of the local economy, which has been hindering, due to the progressive siltation of the canal, making difficult to navigate the boats. This research aimed to analyze and evaluate the influence of flood tide on sedimentation and erosion rate within the channel, in order to assist management policies, raising aspects such as current speed and direction, material in suspension and morphology of channel sections. For that, measurements were made on the channel in a six-hour shift during the flood period, with sample of suspended material, regular channel depth level readings and current data acquisition. Given the samples and field observations, the researchers calculated the liquid and solid canal flow corresponding to the period, demonstrating the intense transport dynamics of suspended sediments in the channel. The results confirmed the strong sedimentary dynamics of the suspended material and the characterization of the local hydrodynamic behavior under the influence of the tide. Therefore, the results support research for the management of future coastal works, in order to remedy the continuous siltation of the channel that directly affects the economic and social activities of the region.
\end{abstract}

Keywords: Tidal depositional systems, Tidal Channel, Currentometry; Suspended Material, Icapuí

\section{INTRODUÇÃO}

Os canais de maré são caracterizados por apresentar apenas um agente modelador: a maré, que propicia o fluxo bidirecional no canal e pela comum associação à três características morfológicas: planícies e barras arenosas não vegetadas; bancos lamosos vegetados e canais configurados em situação discordante às duas feições acima (Davis \& Dalrymple, 2012). Devido a consecutiva ação das marés, os ambientes em questão organizam-se em condições rasas que periodicamente são inundadas, no entanto, os canais podem apresentar dinâmica distinta da planície ao seu redor, onde o transporte sedimentar pode propiciar a erosão ou deposição dos sedimentos.

A remobilização do material em suspensão (MES) apresenta grande relevância nos fenômenos modificadores da morfologia e configuração dos ambientes sedimentares, onde os canais de maré apresentam uma tendência em aprisionar quantidades relativamente altas de areais (Vital et. al., 2008 apud Dean \& Dalrymple, 2002). No entanto, poucos trabalhos abordando essa temática foram desenvolvidas no Brasil, principalmente em canais de maré.

O município de Icapuí tem sua economia fortemente relacionada à região costeira, englobando atividades turísticas, pesqueiras, salineiras e carcinicultura sensivelmente dependentes da flutuação do nível do mar. Especialmente no Porto da Barra Grande, há a presença de diversos barcos pesqueiros de pequeno a médio porte, que usam o canal como ancoradouro e estaleiro. Contudo, relatos da comunidade destacam o progressivo 
assoreamento do canal, dificultando a circulação das embarcações, o que prejudica as atividades locais.

Dessa forma, verificou-se a necessidade de um estudo sobre a remobilização dos sedimentos suspensos diante a dinâmica da maré em seu período de enchente. Para isso, foram coletadas amostras, de material em suspensão, em intervalos de uma hora em um período de seis horas, completando um período entre a maré mínima e a maré máxima durante a sizígia, concomitante a uma coleta de dados através do correntógrafo. Assim, assegurou-se dados para auxiliar na dinâmica e na configuração morfológica e sedimentar da área durante o período.

Além da relevância científica, é importante ressaltar a importância do estudo para futuras decisões sob a gestão costeira, com a finalidade de conter a entrada de sedimentos no canal, a fim de sanar problemáticas que estão ocorrendo no local e que afeta socialmente e economicamente a comunidade, sobretudo a atividade pesqueira.

\section{ÁREA DE ESTUDO}

Localizado na Praia de Requenguela, pertencente ao município de Icapuí -CE, o Porto de Barra Grande é utilizado como ancoradouro e como estaleiro durante os períodos de maré baixa. Atividades salineiras e de carcinicultura também são presentes nas proximidades do canal, no qual é usado para descarregar o rejeito hídrico e como fonte de abastecimento hídrico, respectivamente para o esvaziamento e enchimento dos tanques.

Caracterizado por pequena extensão, três braços e aproximadamente 5 quilômetros de canais, com distância entre as margens de 5 a 100 metros, o canal está inserido em região de mangue e delimitado a norte por flechas arenosas, sua profundidade possui máxima de $3,20 \mathrm{~m}$ e mínima de $0,20 \mathrm{~m}$ em suas extremidades.

A área de estudo (Fig. 1), pertencente ao município de Icapuí é situada a $3 \mathrm{~km}$ do centro da cidade e a $200 \mathrm{~km}$ de Fortaleza, próximo ao restaurante local João Velho e apresenta aproximadamente $4 \mathrm{~km}^{2}$ de abrangência total. Devido à proximidade do município de Icapuí com o Estado do Rio Grande do Norte, o Porto de Macau apresenta-se mais próximo, em comparação com os demais portos da região, sendo assim, usado as informações náuticas do local em questão. 


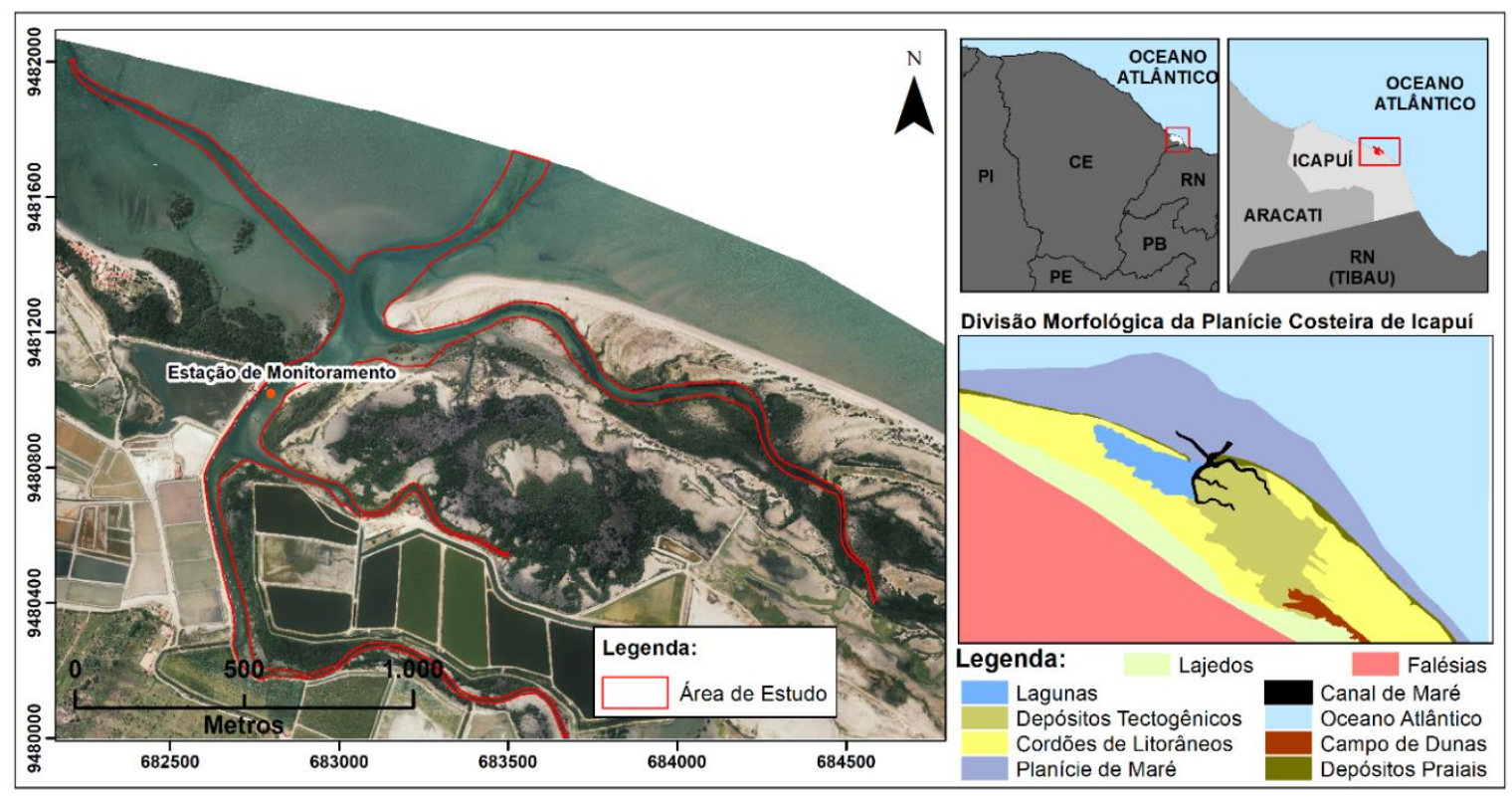

Figura 1: Área de estudo delimitado pela linha vermelha e sua localização (elaborado pelos autores).

\section{MATERIAS E MÉTODOS}

\section{Coleta de dados}

O canal é caracterizado pela grande heterogeneidade nas suas feições nas quais, segundo Davis \& Dalrymple (2012) apresenta planícies, barras arenosas e bancos lamosos vegetados e não vegetados. Apresenta ainda profundidades entre 3 a 0,2 metros e distância entre as margens de $5 \mathrm{~m}$ a $100 \mathrm{~m}$. Dessa maneira, a fim da aquisição de dados mais representativos a estação de monitoramento foi montada na porção de maior profundidade do canal e mais central. Perpendicular às margens e tangente ao ponto de monitoramento foi levantado as cotas altimétricas e calculado a seção molhada, além da instalação do correntógrafo e das coletas do material em suspensão. Para isso, foram observadas imagens de satélites (Quickbird) georeferenciadas, constatando a inundação da área permanente diante os ciclos da maré.

A variação do nível d'água local foi monitorada através de uma régua graduada em $3.10 \mathrm{~m}$ fixada no ponto médio do canal e as leituras ocorreram durante um período de seis horas no intervalo de uma hora, permitindo assim o total de seis leituras.

O correntógrafo usado foi o ACMPLUS 3D (3-Dimensional Acoustic Current Meter) da marca FALMOUTH SCIENTIFIC. O aparelho ficou ativado por seis horas contínuas e adquiriu dados em três dimensões através de quatro transdutores acústicos. $\mathrm{O}$ que possibilitou a leitura de ondas que interceptam o material em suspensão no fluído e calculam a intensidade da corrente bem como a sua direção.

Para a coleta do material em suspensão (MES), foi usado a Garrafa de Van Dorn com capacidade de 5L, após a coleta a cada hora, o material adquirido foi armazenado em garrafas plásticas. Em laboratório, fez-se por meio de membranas de éster com porosidade de $0,45 \mu \mathrm{ma}$ a filtragem do material coletado, com a ajuda de uma bomba a vácuo até atingir a saturação da 
membrana. Desse modo, com o peso inicial e final, após a filtragem, têm-se a quantidade de material retido e o volume de água utilizado, quantificando assim o MES daquele instante.

\section{Processamento dos dados e resultados prévios}

O trabalho consiste na junção de três grandezas: cálculo do perfil molhado, dados correntométricos $\mathrm{e}$ quantidade de material em suspensão. A partir do cálculo do perfil, aliado aos dados adquiridos pelo correntógrafo obtemos a vazão líquida (Vl) daquele momento, que ao multiplicar pela quantidade de material em suspensão resultou na vazão sólida (Vs).

Perfis de quatro momentos distintos foram elaborados a partir das medidas obtidas com a leitura da régua de maré e com o GPS. Desse modo, obteve-se as alturas de maré e os respectivos comprimentos entre as margens do canal. O primeiro momento, compreende a maré local entre $1,2 \mathrm{~m}$ e $1,9 \mathrm{~m}$ e uma distância entre as margens de $20 \mathrm{~m}$ a $35 \mathrm{~m}$; o segundo varia entre $1,9 \mathrm{~m}$ a $2,5 \mathrm{~m}$ de maré e um comprimento de $35 \mathrm{~m}$ a $48 \mathrm{~m}$ entre as margens; o terceiro entre $2,5 \mathrm{~m}$ e $2,95 \mathrm{~m}$ de maré e $48 \mathrm{~m}$ a $57 \mathrm{~m}$ entre as margens. Com os valores obtidos construiu-se perfis que resultaram em áreas mínimas e máximas referentes a cada momento, sendo elas: $14,418 \mathrm{~m}^{2} ; \quad 48,175 \mathrm{~m}^{2} ; \quad 79,675 \mathrm{~m}^{2} \quad \mathrm{e}$ $120,979 \mathrm{~m}^{2}$ (Tabela 1).

Os dados obtidos pelo correntógrafo encontram-se em $\mathrm{m} / \mathrm{s}$, já que é uma medida de velocidade de um corpo, no caso a água. Os valores encontrados, mostram-se análogos ao fluxo da maré observado no canal durante o monitoramento. A maré se inicia com pouca velocidade e tende a um acréscimo repentino e rápido quando se encontra a $2,4 \mathrm{~m}$ de altura, já em sua máxima o fluxo perde velocidade até uma estagnação da maré, subsequente ocorre uma inversão do sentido caracterizado como o início do período de vazante. As médias obtidas, referentes as seis horas de monitoramento, foram respectivamente as seguintes: $0,952 \mathrm{~m} / \mathrm{s}$; $0,356 \mathrm{~m} / \mathrm{s} ; 2,61 \mathrm{~m} / \mathrm{s} ; 2,75 \mathrm{~m} / \mathrm{s} ; 2,3 \mathrm{~m} / \mathrm{s}$ e $1,089 \mathrm{~m} / \mathrm{s}$ (Tabela 1). Com isso calculou a partir da fórmula I a vazão líquida (Vl) obtida em $\mathrm{m}^{3}$. $\mathrm{s}^{-1}$ que é o produto entre a corrente (C) e a área da seção (A) naquela dada hora $(\mathrm{h})$.

$\mathrm{Vl}_{(\mathrm{h})}=\mathrm{C}_{(\mathrm{h})} \times \mathrm{A}_{(\mathrm{h})}(\mathrm{I})$

$\mathrm{Vs}_{(\mathrm{h})}=\mathrm{Vl}_{(\mathrm{h})} \times \mathrm{MES}_{(\mathrm{h})}(\mathrm{II})$

Tabela 1 -tabela com os dados adquiridos (maré, área, corrente, material em suspensão, vazão líquida e vazão sólida) durante o período de seis horas. (elaborado pelos autores)

\begin{tabular}{|c|cccccc|}
\hline Hora $(\mathbf{h})$ & Maré $(\mathbf{m})$ & Área $\left(\mathbf{m}^{\mathbf{2}}\right)$ & Corrente $(\mathbf{m} / \mathbf{s})$ & MES (mg/l) & Ql $\left(\mathbf{m}^{\mathbf{3}} / \mathbf{s}\right)$ & Qs (kg/s) \\
\hline $\mathbf{1 3 : 0 0}$ & 1,2 & 14,42 & 0,95 & 17,06 & 13,73 & 0,23 \\
$\mathbf{1 4 : 0 0}$ & 1,19 & 14,42 & 0,36 & 22,75 & 5,13 & 0,12 \\
$\mathbf{1 5 : 0 0}$ & 1,18 & 14,42 & 2,61 & 23,48 & 37,63 & 0,88 \\
$\mathbf{1 6 : 0 0}$ & 2,4 & 48,18 & 2,76 & 18,79 & 132,72 & 2,49 \\
$\mathbf{1 7 : 0 0}$ & 2,9 & 120,98 & 2,3 & 16,35 & 278,25 & 4,55 \\
$\mathbf{1 8 : 0 0}$ & 2,95 & 120,98 & 1,09 & 16,04 & 131,75 & 2,11 \\
\hline
\end{tabular}


A quantidade de material sólido apresentou uma variação crescente com o aumento da corrente durante as três primeiras horas, seguida por uma decrescência. Os valores foram expressos em $\mathrm{kg} / \mathrm{m}^{3}$ e apresentaram uma variação entre 0,017 a 0,023 no decorrer do período analisado. A partir da quantificação do material sólido foi possível obter o cálculo da vazão sólida (Vs), representada pela fórmula II, que consiste no produto entre a vazão líquida (Vl) e o material em suspensão (MES) naquela hora $(\mathrm{h})$, em $\mathrm{kg} . \mathrm{s}^{-1}$.

\section{RESULTADOS}

Após as coletas de dados e seus respectivos processamento e análises, foi possível gerar um gráfico simples (Fig. 2) no qual apresenta a maré regional, extraída da Tábua de Maré de Macau, $\mathrm{RN}$ e da maré local, Porto da Barra Grande, ambas observadas no dia 10 de outubro de 2018 entre as $13 \mathrm{~h}$ e $18 \mathrm{~h}$ com intervalos de uma hora. Comparando os gráficos pode-se observar um atraso de maré, em aproximadamente uma hora, aonde a linha referente a regional (linha preta) teve variação de $0,45 \mathrm{~m}$ entre as $13 \mathrm{~h}$ e $14 \mathrm{~h}$ e a local (linha cinza) se apresenta contínua, havendo variação somente a partir das $14 \mathrm{~h}$. No período final da enchente vê-se uma estabilização da maré local antecipada à estabilização regional, demonstrando a influência máxima na qual a maré tem sob a variação do nível de maré local. Além disso, é notório a maior variação da maré local quando comparada a regional, tal fenômeno se deve à morfologia do canal apresentar baixa extensão e pequena largura, tornando-o mais sensível à variação de maré diante a sua profundidade.

Através dos dados coletados, foi possível calcular a vazão sólida pertencente a cada período observado, quantificando assim o material em suspensão remobilizado diante às variações da maré no período de enchente. $\mathrm{O}$ resultado é demonstrado na figura 3 e é expresso em $\mathrm{kg} . \mathrm{s}^{-1}$

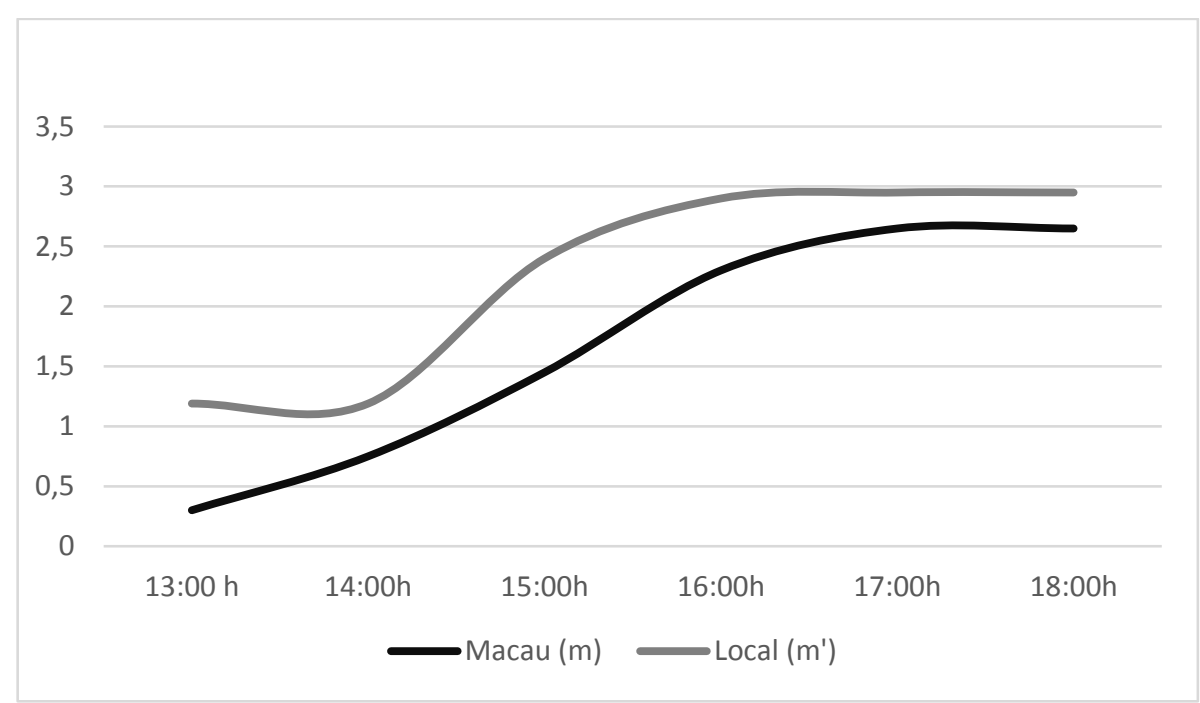

Figura 2: Gráfico comparativo entre maré regional e local (elaborado pelos autores) 


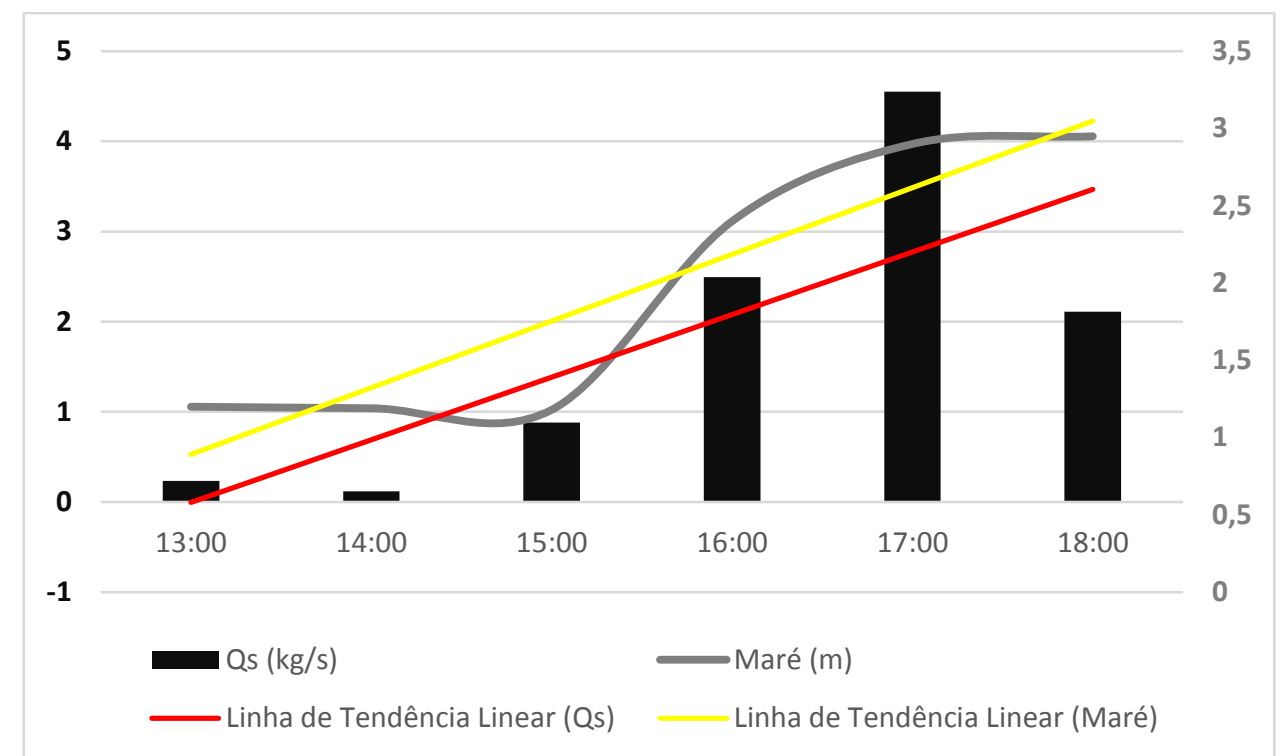

Figura 3: Gráfico vazão sólida (Qs) e maré local e respectivas linhas de tendência (elaborado pelos autores)

Observa-se assim a remobilização ligada diretamente ao avanço da maré, iniciando nas duas primeiras horas com uma baixa vazão sólida, na média de $0,20 \mathrm{~kg} . \mathrm{s}^{-1}$, havendo nas três horas subsequentes um grande aumento até alcançar a vazão máxima de $4.55 \mathrm{~kg} . \mathrm{s}^{-1}$ correspondente a $5^{\mathrm{a}}$ hora do período, que equivale também a maior maré local da área. Após a essa máxima o valor da vazão sólida sofre uma rápida regressão, atingindo os $2,11 \mathrm{~kg} . \mathrm{s}^{-1}$, período no qual a maré estabiliza juntamente com a velocidade, interferindo assim na vazão liquída e sólida.

A ascendência da maré no canal, faz com que a área da sessão molhada aumente significativamente já que o volume é uma variável exponencial $\left(\mathrm{m}^{3}\right)$. Assim, possibilita uma maior área de contato da água com os sedimentos, o que interfere diretamente no cálculo da vazão sólida. Dessa forma, a maré é a variável de maior importância para a dinâmica do material remobilizado, que mais uma vez é comprovada pelas linhas de tendência da vazão sólida e da maré geradas no figura 4 , nas quais se mantêm com a mesma inclinação e lineamento perfazendo as condições estatísticas para a correlação direta entre ambas.

A corrente, é um fator imprescindível para a remobilização do material, porém é uma variável de menor influência quando comparada com a variação do volume gerado pelo avanço da maré. Tal fato é justificado pela corrente se tratar de uma variável não exponencial quando aplicada à fórmula , é possível ver tal relação na figura 05 , no qual há momentos em que a corrente diminui consideravelmente e a vazão sólida se mantêm no topo, momento este que coincide com o máximo da maré local. 


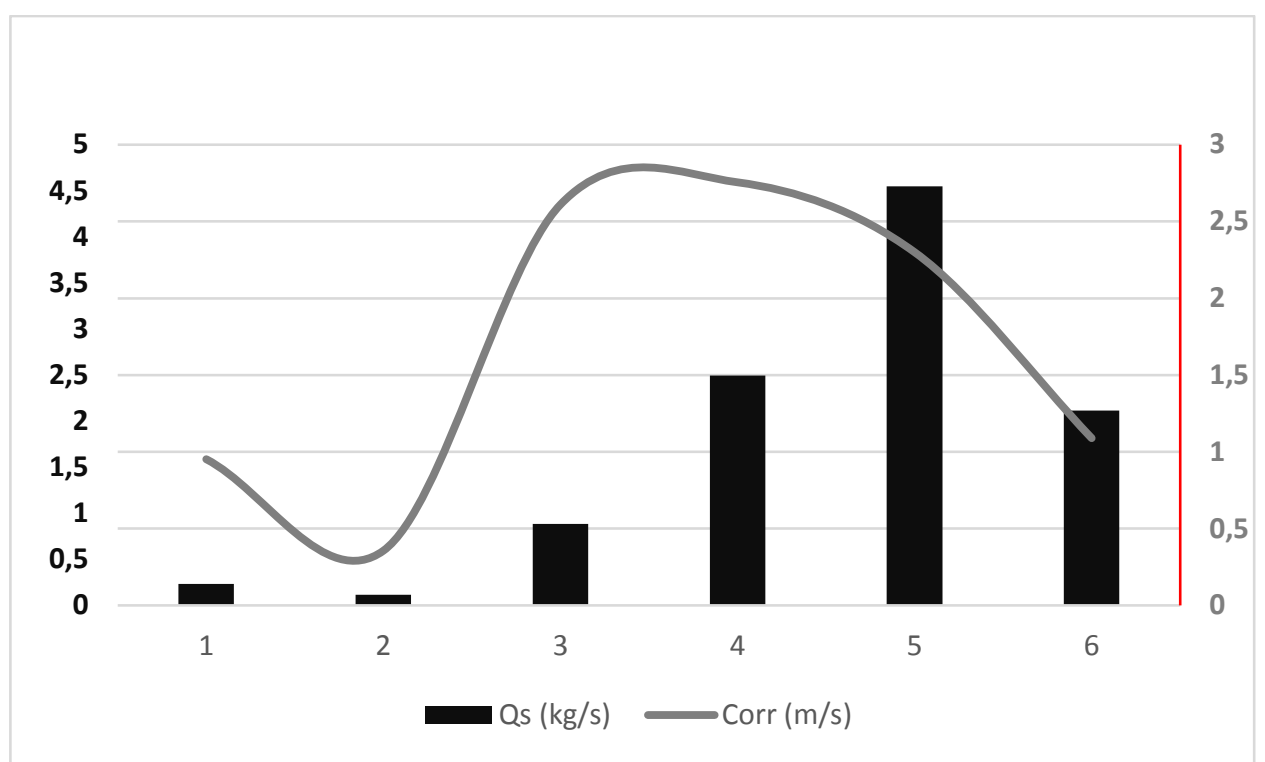

Figura 4: Gráfico da vazão sólida e velocidade da corrente (elaborado pelos autores)

\section{CONCLUSÕES}

Através das análises dos dados obtidos nesse trabalho, foi possível ressaltar a importância da maré sob as variáveis hidrodinâmicas que ocorrem no canal do porto da Barra Grande. A maré é o único agente hídrico no canal, relacionando assim diretamente com a remobilização dos sedimentos ao longo da área. Com a medição da profundidade local viu-se que a maré age em tempos e variações distintas, mostrando que o canal altera sua profundidade 30 minutos após o início da enchente da maré regional extraída da tábua de maré fornecida pelo Centro de Hidrografia da Marinha.

A partir dos cálculos da vazão líquida e sólida, o canal mostrou uma alta quantidade de material remobilizado, chegando a máxima de $4,55 \mathrm{~kg} . \mathrm{s}^{-1}$, condizendo com a intensa dinâmica que sofre o leito do canal relatada pela comunidade local. Verificou-se que o maior agente remobilizante é a maré, devido à sua interferência no cálculo da vazão líquida agir como uma variável exponencial. A corrente, apesar de ser uma variável imprescindível, corrabora em menor expressão nos cálculos das equações.

Dessa forma, constatou a alta quantidade de sedimentos carreados para dentro do canal durante o período de enchente da maré, o que fornece dados iniciais para um estudo de possíveis obras a serem feitas na região para conter o assoreamento do canal. Ainda demonstra a dinâmica do nível d'agua no canal de acordo com o avanço da maré, além da influência dos agentes remobilizantes sob o material em suspensão.

\section{REFERÊNCIAS}

Bezerra, R. G, 2006. Hidrodinâmica do estuário do Rio Choró (Cascavel/Beberibe) litoral leste do Estado do Ceará. Dissertação de Mestrado, Pós Graduação em Geologia-UFC.83p.

$\begin{array}{lll}\text { Carvalho, N. } & \text { O. } 2008\end{array}$ Hidrossedimentologia Prática, $2^{\mathrm{a}}$ Edição, Interciência, Rio de Janeiro.

Davis, R. A.; Dalrymple, R. W. 2012. Principles of Tidal Sedimentology. XVI Edition, Springer, Dordrecht. 
Hughes, Z. J. 2012. Tidal channels on tidal flats and marshes. In: Principles of Tidal Sedimentology. Springer, Dordrecht. p.269-300.

KassaS, M. 2002. Coastal Processes with Engineering Applications, by RG Dean and RA Dalrymple, The Environmentalist24.1. p.60-61.
Suguio, K, 2003. Geologia Sedimentar, 1st Edition, Edgard Blucher, Sao Paulo.

VitaL, H.; Neto, F. S; Plácido Junior, J. S. 2008; Morfodinâmica de um Canal de Maré Tropical: Estudo de Caso na Costa Norte Rio Grandense, Nordeste do Brasil. Revista de Gestão Costeira Integrada-Journal of Integrated Coastal Zone Management, v. 8, n. 2 